\title{
Editorial
}

\section{Surgery in Elderly}

\section{Rupesh Mukhia}

Surgery in the Elderly

Associate Professor of Surgery

KIST Medical College, Tribhuwan University.

Surgery for an aging population is a surgical challenge. ${ }^{1}$ As population of elderly people is increasing, so is the surgical conditions in the final years of life. Surgery in elderly is viewed differently especially in the context of decision making and meaningful outcome. British journal of surgery has recently brought out the special issue on surgery in the elderly with thought-provoking articles about medicolegal issues dealing with informed consent, complexity of surgical treatment, ethics in the end of life care and most importantly, independent existence with reference to elderly surgical patient. So what are the factors do your consider "Decision before Incision" in Elderly? Do you think that the concept of dealing with surgical problems in elderly has be standardized or are we just overlooking the issues at personal level ?

The decision making to plan for surgery in elderly has to be balanced over the quality of life and increasing the life expectancy. There is no doubt that the decision making has to be shared between the surgeon, patient and family about the increased risk of post-operative complication. We can't simply let the patient die without surgical intervention when we can do something better. A ray of hope is often enough as a rationale for proceeding with surgical intervention no matter how poor the outcome might be rather than letting the condition get worse. Even then, in situations where surgery is declined, a surgeon can be responsible for managing distressing symptoms as an essential contribution to the decision-making process in a team. ${ }^{2}$

Meaningful outcome depends largely upon how we make the patient fit for surgery. Age-related psycho-physiological changes and co-morbidities affect older people's tolerance to surgery, making this a major life event with grotesque consequences. However advances in surgery and anaesthesia have greatly optimized surgical treatments and outcomes. Preoperative assessment can help to detect highrisk patients at an early stage and employ interventions, including physiotherapy, nutritional, pharmacological and cognitive support, to reduce the risk of postoperative mortality, morbidity and adverse functional outcomes. ${ }^{3}$
So despite of questionable benefit, novel approaches to surgery in the elderly with multi-disciplinary team approach will certainly work in favour of surgeon with promising result.

\section{References}

1. Soreide K., Wijnhoven B.P.L.: Surgery for an ageing population. BJS Jan 2016;103 :e7-e9.

2. J.P.Dunn.Shared decision-making for the elderly patient with a surgical condition. Br J Surg. 2016 Jan;103(2):e19-20. doi: 10.1002/bjs.10076.

3. E. H. J. Hulzebos and N. L. U. van Meeteren; Making elderly fit for surgery .BJS Jan 2016;103 :e12-e15. 